\title{
EXERGAMES COMO FERRAMENTA TERAPÊUTICA EM PACIENTES COM PARKINSON: UMA REVISÃO DE LITERATURA
}

\author{
EXERGAMES AS A THERAPEUTIC TOOL IN PATIENTS WITH PARKINSON: \\ A LITERATURE REVIEW
}

\section{Lucas Nunes dos Santos, Andreza Bitu de Matos e Antônio José dos Santos Camurça}

\author{
Centro Universitário Doutor Leão Sampaio
}

\section{RESUMO}

A doença de Parkinson (DP) é crônica, degenerativa e debilitante, resulta em disfunções motoras, que levam à fraqueza muscular, dores e tremores, dificultando a realização de atividades como em andar e levantar de cadeiras. A reabilitação através dos exergames vem ganhando destaque, devido ao seu caráter inovador, descontraído e motivacional, principalmente para pacientes crônicos, que requerem muito tempo de tratamento. Este estudo tem o objetivo de realizar uma revisão bibliográfica sobre os exergames como ferramenta terapêutica em pacientes com DP. Nesta Revisão de Literatura, incluíram-se artigos científicos publicados nos últimos oito anos, disponíveis nas principais bases de dados da área da saúde, usando como critério de inclusão os termos: Doença de Parkinson, Exergames, Equilíbrio Postural. A análise das publicações selecionadas evidenciou a suposta melhora nas atividades de vida diária desses pacientes. Terapia com exergames apresentaram resultados melhores ou semelhantes em relação aos grupos de controle (reabilitação tradicional), sejam nas funções motoras ou cognitivas. Evidências indicam que as terapias baseadas em exergames podem ser consideradas como viáveis, seguras e pelo menos tão eficaz quanto a reabilitação tradicional da DP, pois têm capacidade de aumentar a aderência aos exercícios e a confiança no equilíbrio, porém, as evidências são limitadas, pois não há uma padronização dos parâmetros adotados.

Palavras-chave: Doença de Parkinson; Realidade Virtual; Equilíbrio Postural.

\section{ABSTRACT}

The Parkinson's disease (PD) is chronic, degenerative and debilitating, resulting in motor disorders, which lead to muscle weakness, pain and tremors, making it difficult to carry out activities such as walking and standing up from chairs. Rehabilitation through exergames has gained prominence due to its innovative, relaxed and motivational character, especially for chronic patients, who require a long time of treatment. This study aims to carry out a literature review on exergames as a therapeutic tool in PD patients. This Literature Review included scientific articles published in the last eight years, available in the main databases in the health area, using as inclusion criteria the terms: Parkinson's Disease, Exergames, Postural Balance. The analysis of selected publications evidenced the supposed improvement in these patients activities of daily living. Exergame therapy showed better or similar results compared to control groups (traditional rehabilitation), either in motor or cognitive functions. Evidence indicates that exergames based therapies can be considered viable, safe and at least as effective as traditional PD rehabilitation, as they have the ability to increase exercise adherence and confidence in balance, however, the evidence is limited because there is no standardization of the parameters adopted.

Keyword: Parkinson's disease; Virtual Reality; Postural Balance. 


\section{V.10 N.1 (2022) ISSN: 2317-434X}

\section{INTRODUÇÃO}

A doença de Parkinson (DP) é uma doença crônica, degenerativa e debilitante, resulta em disfunções motoras, que leva a fraqueza muscular, dor e tremores, dificultando a realização de atividades como em andar, levantar de cadeiras, lentidão nos movimentos e um declínio nas atividades de vida diária. A DP é a segunda doença neurodegenerativa mais comum em todo o mundo, acomete aproximadamente $1 \%$ de todos os idosos com 65 anos ou mais. Afeta a função neurofisiológica, motora e qualidade de vida (ARAÚJO et al., 2014).

Disfunção no equilíbrio e instabilidade postural são os sintomas incapacitantes mais comuns da DP, causam alterações no controle postural e dificuldade para realizar movimentos voluntários. Quando não tratados podem levar ao aumento da frequência de quedas e lesões, aumentando as chances de desenvolver comorbidade e deficiência (KLAMROTH et al., 2016).

Estudos apontam que $68,3 \%$ das pessoas com DP caem pelo menos uma vez por ano, enquanto $50,5 \%$ caem de forma recorrente (duas ou mais quedas por ano) e $31 \%$ relatam que quase caíram nos últimos 12 meses (LINDHOLM et al., 2015).

Exergame, é um termo usado para descrever jogos eletrônicos que requerem que os jogadores se movam fisicamente em resposta às demandas do jogo, a partir de estímulos sensoriais (geralmente construído a partir de um sensor que registra os movimentos e características físicas do jogador). A grande variedade de exergames disponíveis comercialmente e o escopo dos níveis de intensidade dos exergames permitem que as intervenções sejam adaptadas a aspectos específicos de equilíbrio do paciente. Exergames tem o potencial de facilitar o tratamento nas disfunções do equilíbrio, incluindo a redução da variabilidade do centro de gravidade para pessoas em recuperação de lesão da medula espinhal, lesão cerebral ou acidente vascular cerebral que apresentam graves problemas de equilíbrio (HARRIS et al., 2015).

Este estudo tem o objetivo de realizar uma revisão bibliográfica sobre os exergames como ferramenta terapêutica em pacientes com DP.

\section{METODOLOGIA}

Os exergames acerca do tratamento em pessoas com Parkinson é tratado em vários artigos científicos. A partir disso, este estudo traz uma revisão bibliográfica, um método de pesquisa que se fundamenta em obras já publicadas com evidências científicas comprovadas (BIREME; OPAS; OMS, 2017).

A busca foi executada em bases de dados científicas gratuitas tais como PEDro, SciELO, PubMed e BVS, englobando artigos publicados nos últimos oito anos (2014 a 2021), em português e inglês. Para seleção de artigos foram usados os seguintes termos como critério de inclusão: Doença de Parkinson, Exergames, Equilíbrio Postural. Foram selecionados 42 artigos que abordam os exergames como método terapêutico, especificamente em pacientes com Parkinson.

\section{RESULTADOS E DISCUSSÃO}

A análise das 42 publicações selecionadas evidenciou a suposta melhora nas atividades de vida diária desses pacientes. Nos ensaios clínicos randomizados, os exergames apresentaram resultados melhores ou semelhantes em relação aos grupos de controle (reabilitação tradicional), sejam nas funções motoras (locomoção, estabilização postural e manipulação de objetos) ou cognitivas (atenção, alerta, memória, função executiva) (HUNG et al., 2014; PACHOULAKIS; PAPADOPOULOS; ANALYTI, 2018; SCHAEFFER et al., 2019).

Foram observadas várias deficiências nos estudos clínicos randomizados, dentre elas está a falta de resultados padronizados, de padrões nos métodos utilizados para avaliação do paciente e ausência de protocolos de acompanhamento. Também foi observado que na maior parte das publicações, o uso do sensor Kinect da Microsoft para reabilitação da DP foi o mais utilizado (36 de 42 publicações mencionam o Kinect), quando comparado aos outros sensores (Wii Remote, PlayStation Move, óculos VR e afins). Além disso, em artigos recentes, também foi verificado um interesse no desenvolvimento de métodos de reabilitação que possam ser realizados em um cenário de uso doméstico monitorado à distância como continuidade ao tratamento clínico (FERRAZ et al., 2018; SONG et al., 2018; TOLLAR; NAGY; HORTOBÁGYI, 2019).

Nos estudos de casos, os exergames demonstraram resultados significativos em termos de usabilidade e segurança, mesmo com a falta de um grupo de controle, randomização e/ou falta de detalhes das sessões e dados (CIKAJLO et al., 2018; NUIC et al., 2018).

No geral, nenhum dos estudos mostraram que os exergames eram piores do que a terapia de reabilitação tradicional.

\section{CONSIDERAÇÕES FINAIS}

Evidências indicam que as terapias baseadas em exergames podem ser consideradas como viáveis, seguras 


\section{V.10 N.1 (2022) ISSN: 2317-434X}

e pelo menos tão eficaz quanto a reabilitação tradicional da Doença de Parkinson, pois têm capacidade de aumentar a aderência aos exercícios e a confiança no equilíbrio, porém, as evidências são limitadas, pois não há uma padronização dos parâmetros adotados. A falta de resultados padronizados nesta área de pesquisa torna difícil a possibilidade de determinar qual plataforma e método de aplicação dos exergames funcionam melhor para as terapias de reabilitação dos pacientes com DP. É cedo para determinar de forma quantitativa o efeito da terapia com exergames, seja na melhora ou no mantimento da funcionalidade. Por isso, são necessários estudos experimentais em maiores escalas, além de estudos observacionais para avaliar os efeitos motores da terapia baseada em exergames a longo prazo. Mas, é notável os avanços recentes nesta área nos últimos anos.

\section{REFERÊNCIAS}

ARAÚJO, M. et al. Realidade virtual: efeitos na recuperação do membro superior de pacientes hemiparéticos por acidente vascular cerebral. ACM arq. catarin. med, v. 43, n. 1, p. 15-20, 2014.

BIREME; OPAS; OMS. Tipos metodológicos de estudo. p. $5,11,2017$.

CIKAJLO, I. et al. Can telerehabilitation games lead to functional improvement of upper extremities in individuals with Parkinson's disease? International Journal of Rehabilitation Research, v. 41, n. 3, p. 230 238, 2018.

FERRAZ, D. D. et al. The Effects of Functional Training, Bicycle Exercise, and Exergaming on Walking Capacity of Elderly Patients With Parkinson Disease: A Pilot Randomized Controlled Single-blinded Trial. Archives of Physical Medicine and Rehabilitation, v. 99, n. 5, p. 826-833, 2018.

HARRIS, D. M. et al. Exergaming as a viable therapeutic tool to improve static and dynamic balance among older adults and people with idiopathic Parkinson's disease: A systematic review and meta-analysis. Frontiers in Aging Neuroscience, v. 7, n. SEP, 2015.

HUNG, J. W. et al. Randomized comparison trial of balance training by using exergaming and conventional weight-shift therapy in patients with chronic stroke. Archives of Physical Medicine and Rehabilitation, v. 95, n. 9, p. 1629-1637, 2014.

KLAMROTH, S. et al. Effects of exercise therapy on postural instability in Parkinson disease: A meta-analysis. Journal of Neurologic Physical Therapy, v. 40, n. 1, p. 3-14, 2016.

LINDHOLM, B. et al. Prediction of falls and/or near falls in people with mild Parkinson's disease. PLoS ONE, v. 10, n. 1, p. 1-11, 2015.

NUIC, D. et al. The feasibility and positive effects of a customised videogame rehabilitation programme for freezing of gait and falls in Parkinson's disease patients: A pilot study. Journal of NeuroEngineering and Rehabilitation, v. 15, n. 1, p. 1-11, 2018.

PACHOULAKIS, I.; PAPADOPOULOS, N.; ANALYTI, A. Kinect-based exergames tailored to Parkinson patients. International Journal of Computer Games Technology, v. 2018, 2018.

SCHAEFFER, E. et al. Effects of exergaming on attentional deficits and dual-tasking in Parkinson's disease. Frontiers in Neurology, v. 10, n. JUN, p. 1-8, 2019.

SONG, J. et al. Home-based step training using videogame technology in people with Parkinson's disease: a singleblinded randomised controlled trial. Clinical Rehabilitation, v. 32, n. 3, p. 299-311, 2018.

TOLLAR, J.; NAGY, F.; HORTOBÁGYI, T. Vastly Different Exercise Programs Similarly Improve Parkinsonian Symptoms: A Randomized Clinical Trial. Gerontology, v. 65, n. 2, p. 120-127, 2019. 